\title{
Influência dos Períodos de Serviço Anterior e Corrente e do Período Seco Anterior sobre a Produção de Leite na Raça Holandesa
}

\author{
Nilson Milagres Teixeira1, José Valente ${ }^{1}$, Rui da Silva Verneque ${ }^{1}$, Ary Ferreira de Freitas ${ }^{1}$
}

\begin{abstract}
RESUMO - O objetivo deste trabalho foi avaliar os efeitos dos períodos de serviço anterior e corrente e do período seco anterior ajustados simultaneamente como efeitos fixos em modelo de touro para produção de leite até 305 dias, contendo ainda os efeitos fixos de rebanho-ano-estação, grupo genético e idade da vaca e os efeitos aleatórios de touro e vaca. Foi usada matriz de parentesco, que incluiu o touro, o seu pai e sua mãe. O mesmo modelo com exclusão da idade da vaca foi usado para produção de leite até 305 dias ajustada à maturidade. As soluções nas análises foram obtidas usando-se o método da máxima verossimilhança restrita e o algoritmo livre de derivada. Polinômios ortogonais foram ajustados para suavização das soluções. Soluções e valores previstos para produção até 305 dias não foram apresentados, por terem seguido mesma tendência da produção até 305 dias ajustada à maturidade. A produção até 305 dias ajustada à maturidade aumentou $950 \mathrm{~kg}$, quando o período de serviço anterior variou de 20 a 300 dias. Este aumento foi de $802 \mathrm{~kg}$ para a mesma variação do período de serviço corrente. Vacas com período seco de 60 a 80 dias obtiveram a produção de leite mais alta na lactação seguinte. Valores estimados da heritabilidade foram 0,02 para o período de serviço e 0,08 para o período seco, quando a produção total foi considerada no modelo, e 0,12 quando foi excluída. Fatores multiplicativos foram estimados para o ajustamento da produção de leite para os efeitos de período de serviço e seco.
\end{abstract}

Palavras-chave: fatores de ajustamento, período de serviço, período seco, produção de leite, raça holandesa

\section{Effects of the Previous and Present Days Open and of the Previous Dry Days Period on the Milk Production of the Holstein Breed}

\begin{abstract}
The objective of this work was to evaluate the effects of previous and present days open and previous dry days period fitted simultaneously as fixed effects using a sire model for 305-day milk production, still containing the fixed effects of herdyear-season, genetic group, age of cow and the random effects of sire and cow. An additive relationship matrix that included the bull, its sire and its mother was used. The same model with the exclusion of the age of cow was used to the milk production adjusted to a 305-day mature equivalent basis The analysis solutions were obtained using derivative free restricted maximum likelihood. Solutions were smoothened with orthogonal polynomials. Predicted values for 305-day milk production were not presented because they followed the same trend as did milk production adjusted to a 305-day mature equivalent basis. As previous and present days open increased from 20 to 300 days, the milk production increased 950 and $802 \mathrm{~kg}$, respectively. Cows with days dry between 60 to 80 days obtained higher milk production in the following lactation. Heritability estimates were .02 for days open and .08 for days dry, when total milk production was considered in the model, and .12 when it was excluded. Multiplicative adjustment factors were developed to adjust production for the effects of days open and days dry.
\end{abstract}

Key Words: adjustment factors, days open, days dry, milk production, Holstein breed

\section{Introdução}

Nas avaliações genéticas de vacas e touros, é necessário que se considerem os fatores de meio ambiente que influem no desempenho dos animais. Ajustamentos para variações atribuídas aos efeitos de rebanho, ano e estação são realizados fazendo-se comparações dentro destas classes, enquanto o ajustamento para a variação atribuída ao período de lactação é feito limitando-se o número de dias em lactação até 305. Variações atribuídas aos efeitos de idade são removidas por fatores de correção, expressando-se as produções na base de equivalência à maturidade.

Vários autores demostraram que a duração do período de serviço possui efeito significativo na produção de leite. A produção elevou-se com o aumento do período de serviço na lactação corrente (OLTENACU et al., 1980; THOMPSON et al., 1982). $\mathrm{Na}$ lactação anterior, os períodos de serviço longos foram responsáveis pelo aumento na produção da lactação corrente (BAR-ANAN e SOLLER, 1979; 
FUNK et al., 1987; e BROTHERSTONE, 1987). Vacas primíparas foram menos influenciadas pelo período de serviço que multíparas (AURAN, 1974; OLTENACU et al., 1980; e THOMPSON et al., 1982), sendo que os efeitos relativos foram semelhantes.

Quando a concepção ocorreu com menos de 100 dias pós-parto (FUNK et al., 1987; OLTENACU et al., 1980) e os períodos secos foram inferiores a 40 dias (FUNK et al., 1987), houve queda da produção na lactação seguinte.

A variação na duração do período de serviço atribuída à variância genética aditiva tem sido baixa, como evidenciado pelas estimativas de heritabilidade inferiores a 10\% (BERGER et al., 1981; THOMPSON et al., 1982; e HANSEN et al., 1983). Em vista da baixa variabilidade genética, o ajustamento da produção para período de serviço tem sido sugerido por vários autores (SCHAEFFER e HENDERSON, 1972; THOMPSON et al., 1982).

Em todos os trabalhos referenciados, os autores utilizaram o método dos quadrados mínimos, com a premissa de amostragem aleatória dos dados. É possível que vacas de segunda ou de lactações subseqüentes tenham sido selecionadas com base na sua produção de leite da lactação anterior. Por esta razão, procedimentos com base no método da máxima verossimilhança têm sido, mais recentemente, usados para minimizar possíveis efeitos da seleção. $\mathrm{O}$ método da máxima verossimilhança restrita (REML) sugerido por PATTERSON e THOMPSON (1971), além disso, leva em conta a perda de graus de liberdade, por causa do ajustamento para efeitos fixos, tendo sido o método preferido para estimação de componentes de variância em trabalhos de melhoramento genético (MEYER, 1987).

Os objetivos do presente trabalho foram verificar a influência dos períodos de serviço anterior e corrente e período seco anterior sobre a produção de leite até 305 dias e estimar heritabilidades dos períodos de serviço e seco.

\section{Material e Métodos}

Os registros de produção e reprodução em rebanhos da raça Holandesa dos Estados de MG, RS, PR, $\mathrm{SC}$ e SP, usados neste estudo, foram obtidos do Arquivo Zootécnico Nacional mantido na Embrapa Gado de Leite. A apresentação de pelo menos 20 lactações foi condição essencial para que um rebanho-ano fosse incluído nas análises. Outras condições para que uma lactação fosse incluída foram: período de lactação de 150 a 450 dias, produção até
305 dias (L305) e produção total de 1000 a $13.000 \mathrm{~kg}$, período de serviço de 20 a 320 dias, intervalo de partos de 280 a 730 dias, período seco de 0 a 400 dias e idade ao parto de 20 a 207 meses. Os registros foram limitados aos das filhas de touros, com mínimo de 10 filhas em pelo menos três rebanhos. Além disso, cada classe de rebanho-ano-estação deveria possuir filhas de pelo menos dois touros e cada vaca, a sua primeira lactação. Após satisfeitas todas as restrições, restaram para as análises 10.708 lactações iniciadas no período de 1981 a 1992, de 4880 vacas filhas de 323 touros. Estas lactações estavam distribuídas em 214 rebanhos e 1571 classes de rebanhoano-estação. As duas estações foram definidas como das águas, o período de outubro a março, e da seca, no período de abril a setembro.

Os períodos de serviço, seguidos por partos normais, foram calculados subtraindo-se o período de gestação (280 dias) do intervalo de partos. Desse modo, as lactações sem registros dos partos subseqüentes foram descartadas nas análises. As produções de leite analisadas foram produção até 305 dias (L305) e produção até 305 dias ajustada a idade adulta (PROAJU), conforme relatado por TEIXEIRA et al. (1992).

O modelo usado na análise de variância de L305 foi um modelo de touro que incluiu os efeitos fixos de rebanho-ano-estação (absorvido), grupo genético (1 - para 31/32 Holandês; 2 - para vacas puras por cruza com geração controlada, GCs; e 3 - para puras de origem), classes de períodos de serviço anterior (PA) e corrente (PS) e período seco anterior (PC), efeito de idade ao parto como covariável até o $2^{\circ}$ grau e os efeitos aleatórios de touro e vaca. Uma matriz de parentesco incluiu o touro, seu pai e sua mãe. As soluções das equações do modelo foram obtidas usando-se o método da máxima verossimilhança restrita por intermédio de algoritmo livre de derivadas (MTDFREML), desenvolvido por BOLDMAN et al. (1995). Este mesmo modelo, excluindo a covariável idade, foi usado para PROAJU.

Para a estimação das heritabilidades dos períodos de serviço e seco, o conjunto de dados usado constituiu-se de 12.080 lactações de filhas de 271 touros, sendo que cada um deveria possuir, no mínimo, 10 filhas em pelo menos três rebanhos. O programa MTDFREML foi também usado. No modelo para estimação da heritabilidade do período seco, foram considerados os efeitos fixos de rebanho-ano-estação (absorvido), grupo genético, as covariáveis (efeitos linear e quadrático) idade ao parto, períodos de serviço anterior e corrente e efeito aleatório de touro. 
Rev. bras. zootec.

Em segundo modelo, além dos efeitos anteriores, incluiu-se também a produção total de leite. O modelo para estimação da heritabilidade do período de serviço incluiu os efeitos fixos de rebanho-ano-estação (absorvido), grupo genético e idade ao parto (lineare quadrático) como covariáveis e efeito aleatório de touro.

Polinômios ortogonais foram usados para suavizar soluções para as classes de períodos de serviço e seco (SAS, 1990). Fatores multiplicativos para ajustamento da produção de leite para classes de duração dos períodos de serviço ou seco foram calculados como:

$$
F_{c}=\frac{\hat{\mu}+\hat{y} b}{\hat{\mu}+\hat{y} k}
$$

em que

$\hat{\mu}$ é a média aritmética de L305 ou PROAJU para todas as lactações;

$\hat{y}$ b é o valor previsto de L305 ou PROAJU para o intervalo de classes para o qual os dados foram ajustados; e

$\hat{\mathrm{y}}_{\mathrm{k}}$ é o valor previsto de L305 ou PROAJU para $\mathrm{o}$ intervalo de classe $\mathrm{k}$.

\section{Resultados e Discussão}

O número de observações por intervalo de classe para período de serviço anterior, período de serviço corrente e período seco encontra-se na Tabela 1 . Soluções para produção de leite até 305 dias e produção de leite até 305 dias ajustada à maturidade pelo método REML, para intervalos de classe de período de serviço anterior, período de serviço corrente e período seco anterior, encontram-se no Tabela 2. Soluções e valores previstos para produção até 305 dias ajustada à maturidade encontram-se representados nas Figuras 1, 2 e 3 . Os valores para produção até 305 dias seguiram a mesma tendência da produção até 305 dias, ajustada à maturidade, razão pela qual não foram representados. Em análises preliminares, VALENTE et al. (1995), usando os mesmos dados e o método dos quadrados mínimos, mostraram que os efeitos do período de serviço anterior, período de serviço corrente e período seco anterior influenciaram $(\mathrm{P}<0,01)$ a produção até 305 dias ajustada à maturidade. A relação entre produção até 305 dias ajustada à maturidade e período de serviço anterior foi linear, semelhante aos resultados obtidos por FUNK et al. (1987) e VALENTE et al. (1995), sendo, entretanto, a magnitude da variação na produção duas vezes maior que a relatada em FUNK et al. (1987). A relação curvilínea entre produção até 305
Tabela 1 - Número de observações para período de serviço anterior e de serviço corrente e período seco anterior, por intervalos de classe

Table 1 - Number of observations for previous days open, present days open and previous days dry, per subclass intervals

\begin{tabular}{|c|c|c|c|}
\hline \multirow{3}{*}{$\begin{array}{l}\text { Intervalo (dias) } \\
\text { Interval (days) }\end{array}$} & \multicolumn{2}{|c|}{$\begin{array}{l}\text { Período de serviço } \\
\text { Days open }\end{array}$} & \multirow{2}{*}{$\begin{array}{l}\text { Período seco } \\
\text { Days dry } \\
\text { Anterior }\end{array}$} \\
\hline & Anterior & Corrente & \\
\hline & Previous & Present & Previous \\
\hline $0-9$ & - & - & 49 \\
\hline $10-19$ & - & - & 101 \\
\hline $20-29$ & 38 & 32 & 154 \\
\hline $30-39$ & 95 & 89 & 325 \\
\hline $40-49$ & 250 & 237 & 716 \\
\hline $50-59$ & 612 & 584 & 1269 \\
\hline $60-69$ & 868 & 880 & 1671 \\
\hline $70-79$ & 1071 & 1022 & 1561 \\
\hline $80-89$ & 1163 & 1125 & 1193 \\
\hline $90-99$ & 983 & 943 & 767 \\
\hline $100-109$ & 800 & 767 & 588 \\
\hline $110-119$ & 669 & 669 & 430 \\
\hline $120-129$ & 585 & 581 & 349 \\
\hline $130-139$ & 515 & 504 & 260 \\
\hline $140-149$ & 425 & 451 & 227 \\
\hline $150-159$ & 364 & 359 & 175 \\
\hline $160-169$ & 294 & 335 & 133 \\
\hline $170-179$ & 284 & 273 & 133 \\
\hline $180-189$ & 249 & 275 & 289 \\
\hline $190-199$ & 212 & 218 & 206 \\
\hline $200-209$ & 199 & 192 & 112 \\
\hline $210-219$ & 150 & 192 & \\
\hline $220-229$ & 129 & 146 & \\
\hline $230-239$ & 124 & 135 & \\
\hline $240-249$ & 100 & 120 & \\
\hline $250-259$ & 96 & 104 & \\
\hline $260-269$ & 96 & 95 & \\
\hline $270-279$ & 77 & 75 & \\
\hline $280-289$ & 77 & 74 & \\
\hline $290-299$ & 60 & 79 & \\
\hline $300-309$ & 58 & 72 & \\
\hline $310-319$ & 65 & 80 & \\
\hline
\end{tabular}

dias ajustada à maturidade e período de serviço corrente foi semelhante à de outros autores (FUNK et al., 1987; SCHAEFFER e HENDERSON, 1972; VALENTE et al., 1995), sendo que a produção aumentou em taxas crescentes com período de serviço corrente até aproximadamente 100 dias, crescendo daí por diante em taxas mais baixas. O efeito do período do serviço corrente sobre a produção de leite foi maior que o efeito do período de serviço anterior (Tabelas 2 e 3), concordando com SADEK e FREEMAN (1992), quando consideraram lactações de todas as ordens.

Vacas com período seco anterior de 60 a 80 dias foram as que produziram mais leite na lactação 
Tabela 2 - Soluções para produção de leite até 305 dias ajustada à maturidade (PROAJU) e produção de leite até 305 dias (L305), para períodos de serviço anterior e corrente e período seco anterior

Table 2 - Solutions for milk production up to 305-day adjusted to the maturity ( PROAJU) and 305-day milk production (L305) for previous and present days open and previous days dry

\begin{tabular}{|c|c|c|c|c|c|c|}
\hline \multirow[t]{3}{*}{$\begin{array}{l}\text { Intervalo (dias) } \\
\text { Interval (days) }\end{array}$} & \multicolumn{4}{|c|}{$\begin{array}{c}\text { Período de serviço } \\
\text { Days open }\end{array}$} & \multicolumn{2}{|c|}{$\begin{array}{l}\text { Período seco } \\
\text { Days dry }\end{array}$} \\
\hline & \multicolumn{2}{|c|}{$\begin{array}{l}\text { Anterior } \\
\text { Previous }\end{array}$} & \multicolumn{2}{|c|}{$\begin{array}{l}\text { Corrente } \\
\text { Present }\end{array}$} & \multicolumn{2}{|c|}{$\begin{array}{l}\text { Anterior } \\
\text { Previous }\end{array}$} \\
\hline & $\overline{\text { PROAJU }}$ & L305 & PROAJU & L305 & PROAJU & L305 \\
\hline $0-9$ & - & - & - & - & $-486,18$ & $-446,53$ \\
\hline $10-19$ & - & - & - & - & $-757,57$ & $-697,12$ \\
\hline $20-29$ & $-307,43$ & $-245,92$ & $-698,69$ & $-648,60$ & $-672,02$ & $-618,30$ \\
\hline $30-39$ & $-270,59$ & $-226,96$ & $-946,73$ & $-894,89$ & $-514,34$ & $-463,85$ \\
\hline $40-49$ & $-323,96$ & $-302,81$ & $-758,14$ & $-715,74$ & $-299,30$ & $-261,48$ \\
\hline $50-59$ & $-119,19$ & $-104,94$ & $-561,27$ & $-533,25$ & $-24,20$ & $-25,38$ \\
\hline $60-69$ & $-117,67$ & $-115,34$ & $-436,91$ & $-427,38$ & 0,00 & 0,00 \\
\hline $70-79$ & $-28,25$ & $-28,83$ & $-298,18$ & $-289,45$ & 32,70 & 30,03 \\
\hline $80-89$ & 0,00 & 0,00 & $-193,70$ & $-193,65$ & $-47,13$ & $-44,37$ \\
\hline $90-99$ & 32,99 & 32,15 & $-131,35$ & $-129,90$ & $-27,52$ & $-29,99$ \\
\hline $100-109$ & $-20,54$ & $-24,11$ & 13,26 & $-6,08$ & $-72,68$ & $-72,48$ \\
\hline $110-119$ & 151,76 & 144,95 & $-17,07$ & $-36,31$ & $-84,92$ & $-96,25$ \\
\hline $120-129$ & 49,86 & 45,11 & $-6,29$ & $-13,03$ & $-18,88$ & $-16,43$ \\
\hline $130-139$ & 131,30 & 138,97 & 71,89 & 76,36 & $-186,96$ & $-180,37$ \\
\hline $140-149$ & $-2,58$ & $-0,42$ & $-6,68$ & $-6,61$ & $-170,10$ & $-165,50$ \\
\hline $150-159$ & 93,87 & 98,03 & 42,45 & 17,76 & $-174,86$ & $-188,21$ \\
\hline $160-169$ & 165,12 & 158,35 & 88,45 & 70,94 & $-285,61$ & $-267,71$ \\
\hline $170-179$ & 117,21 & 129,66 & 45,05 & 45,00 & $-342,23$ & $-320,68$ \\
\hline $180-189$ & 203,76 & 203,63 & 0,00 & 0,00 & $-205,16$ & $-195,85$ \\
\hline $190-199$ & 132,37 & 146,17 & 86,44 & 88,43 & $-496,26$ & $-479,59$ \\
\hline $200-209$ & 428,67 & 419,88 & 61,90 & 55,81 & $-337,06$ & $-340,73$ \\
\hline $210-219$ & 354,48 & 359,41 & 99,11 & 89,67 & & \\
\hline $220-229$ & 412,11 & 402,08 & 128,74 & 117,10 & & \\
\hline $230-239$ & 208,66 & 214,18 & 257,08 & 222,07 & & \\
\hline $240-249$ & 465,02 & 455,75 & 145,18 & 147,67 & & \\
\hline $250-259$ & 560,62 & 553,02 & 21,44 & 14,70 & & \\
\hline $260-269$ & 377,33 & 382,67 & 3,64 & 22,02 & & \\
\hline $270-279$ & 532,64 & 487,27 & 52,38 & 45,88 & & \\
\hline $280-289$ & 701,05 & 685,81 & 197,48 & 221,30 & & \\
\hline $290-299$ & 645,57 & 672,90 & 103,11 & 88,08 & & \\
\hline $300-309$ & 642,14 & 648,24 & $-32,70$ & $-21,43$ & & \\
\hline $310-319$ & 550,93 & 557,46 & 68,64 & 64,83 & & \\
\hline
\end{tabular}

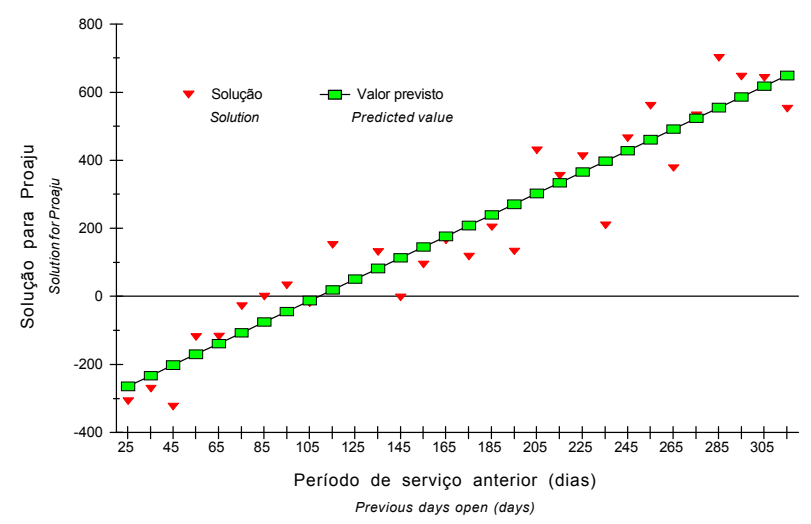

Figura 1 - Relação entre produção de leite até 305 dias ajustada à maturidade (PROAJU) e período de serviço anterior.

Figure 1 - Relationship between milk production up to 305-day adjusted to the maturity (PROAJU) and previous days open.

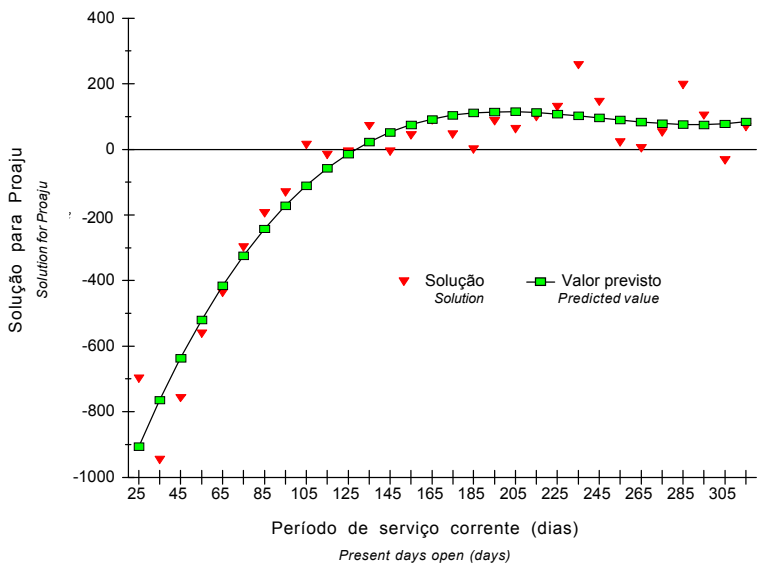

Figura 2 - Relação entre produção de leite até 305 dias ajustada à maturidade (PROAJU) e período de serviço corrente.

Figure 2 - Relationship between milk production up to 305-day adjusted to the maturity (PROAJU) and present days open. 
Rev. bras. zootec.

Tabela 3 - Fatores multiplicativos para períodos de serviço anterior e corrente e período seco anterior para produção até 305 dias ajustada à maturidade (PROAJU)

Table 3 - Multiplicative factors for previous and present days open and previous days dry for production up to 305day adjusted to the maturity (PROAJU)

\begin{tabular}{|c|c|c|c|}
\hline \multirow[t]{2}{*}{$\begin{array}{l}\text { Intervalo (dias) } \\
\text { Interval (days) }\end{array}$} & \multicolumn{2}{|c|}{$\begin{array}{l}\text { Período de serviço } \\
\text { Days open }\end{array}$} & \multirow[t]{2}{*}{$\begin{array}{c}\text { Período seco } \\
\text { Days dry }\end{array}$} \\
\hline & $\begin{array}{l}\text { Anterior } \\
\text { Previous }\end{array}$ & $\begin{array}{c}\text { Corrente } \\
\text { Present }\end{array}$ & \\
\hline$\overline{0-9}$ & - & - & 1,08 \\
\hline $10-19$ & - & - & 1,06 \\
\hline $20-29$ & 1,04 & 1,14 & 1,05 \\
\hline $30-39$ & 1,04 & 1,12 & 1,03 \\
\hline $40-49$ & 1,03 & 1,09 & 1,02 \\
\hline $50-59$ & 1,03 & 1,07 & 1,01 \\
\hline $60-69$ & 1,02 & 1,06 & 1,00 \\
\hline $70-79$ & 1,02 & 1,04 & 0,99 \\
\hline $80-89$ & 1,01 & 1,03 & 0,99 \\
\hline $90-99$ & 1,01 & 1,02 & 0,98 \\
\hline $100-109$ & 1,00 & 1,01 & 0,98 \\
\hline $110-119$ & 1,00 & 1,00 & 0,98 \\
\hline $120-129$ & 1,00 & 0,99 & 0,98 \\
\hline $130-139$ & 0,99 & 0,99 & 0,99 \\
\hline $140-149$ & 0,99 & 0,98 & 0,99 \\
\hline $150-159$ & 0,98 & 0,98 & 1,00 \\
\hline $160-169$ & 0,98 & 0,98 & 1,00 \\
\hline $170-179$ & 0,97 & 0,98 & 1,01 \\
\hline 180-189 & 0,97 & 0,98 & 1,02 \\
\hline 190-199 & 0,96 & 0,98 & 1,04 \\
\hline $200-209$ & 0,96 & 0,98 & 1,05 \\
\hline $210-219$ & 0,96 & 0,98 & 1,04 \\
\hline $220-229$ & 0,95 & 0,98 & 1,05 \\
\hline $230-239$ & 0,95 & 0,98 & \\
\hline $240-249$ & 0,94 & 0,98 & \\
\hline $250-259$ & 0,94 & 0,98 & \\
\hline $260-269$ & 0,93 & 0,98 & \\
\hline $270-279$ & 0,93 & 0,98 & \\
\hline $280-289$ & 0,93 & 0,98 & \\
\hline 290-299 & 0,92 & 0,98 & \\
\hline $300-309$ & 0,92 & 0,98 & \\
\hline $310-319$ & 0,92 & 0,98 & \\
\hline
\end{tabular}

seguinte. Estes resultados concordam com os de FUNK et al. (1987), SCHAEFFER e HENDERSON (1972) e MAKUZA e McDANIEL (1996). Períodos secos mais curtos ou muito longos apresentaram efeito prejudicial na produção de leite. Estes resultados estão de acordo com a recomendação geral de manejo de que 60 dias é o ideal para o período seco. Para FUNK et al. (1987), períodos secos mais longos que 60 dias foram moderadamente prejudiciais à produção, enquanto no presente trabalho e no de SCHAEFFER e HENDERSON (1972) houve queda acentuada a partir de 100 dias de período seco.

Os valores previstos foram usados para o cálculo de fatores multiplicativos de ajustamento, sendo os

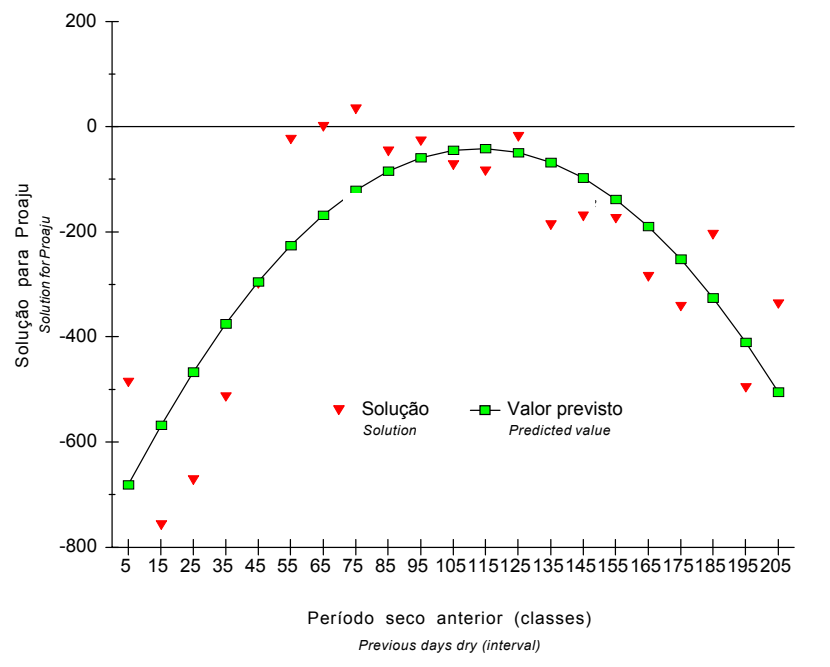

Figura 3 - Relação entre produção de leite em até 305 dias ajustada à maturidade (PROAJU) e período seco anterior.

Figure 3 - Relationship between milk production up to 305-day adjusted to the maturity (PROAJU) and previous days dry.

períodos de serviço anterior e corrente padronizados para o intervalo de 100 a 109 dias e o período seco para 60 a 69 dias. Os fatores para ajustamento da produção até 305 dias, ajustada à maturidade, para períodos de serviço anterior e corrente (Tabela 3), foram semelhantes aos de FUNK et al. (1987), diferindo, respectivamente, de no máximo 0,03 e 0,04, sendo estas diferenças observadas para as classes mais altas do período de serviço. Houve semelhança também dos fatores para período seco anterior, sendo as diferenças maiores para os períodos secos até 30 dias. Os fatores obtidos para período de serviço anterior, em geral, foram da mesma magnitude dos relatados por VALENTE et al. (1995), com diferenças para períodos de serviço inferiores a 60 dias. Fatores para período de serviço corrente diferenciaram até 0,12 para classes mais altas. Neste trabalho, diferenças maiores para período seco anterior também foram obtidas para classes mais altas, assemelhando-se aos resultados de VALENTE et al. (1995),

O valor estimado da heritabilidade do período de serviço foi 0,02 (Tabela 4), sendo semelhante ao encontrado por vários autores que também usaram o modelo de touro (BERGER et al., 1981; HANSEN et al., 1983; e FUNK et. al., 1987). As estimativas da heritabilidade do período seco foram, respectivamente, 0,08 e 0,12 , quando a produção total foi incluída ou excluída como covariável no modelo, demonstrando que a inclusão da produção reduz a variabilidade 
Tabela 4 - Estimativas de componentes de variância e heritabilidade para períodos de serviço e seco Table 4 - Variance components and heritability estimates for days open and days dry

\begin{tabular}{lcccr}
\hline & \multicolumn{3}{c}{ Variância } & \multirow{2}{*}{$\begin{array}{c}\text { Heritabilidade } \\
\text { Heritability }\end{array}$} \\
\cline { 2 - 4 } & $\begin{array}{c}\text { Genética } \\
\text { Genetic }\end{array}$ & $\begin{array}{c}\text { Meio ambiente } \\
\text { Enviromental }\end{array}$ & $\begin{array}{c}\text { Fenotípica } \\
\text { Phenotypic }\end{array}$ & 0,02 \\
Período de serviço $^{1}$ & 19,4888 & 3510,3941 & 3529,8828 & 0,12 \\
Days open & & & & \\
Período seco $^{2}$ & $23,3222^{\mathrm{a}}$ & 788,5291 & 41,8513 & 0,08 \\
Days $d r y$ & $12,0566^{\mathrm{b}}$ & 482,8508 & 494,9074 & \\
\hline
\end{tabular}

${ }_{1}^{1}$ Modelo: $\hat{Y}=\mu+$ rebanho-ano-estação + grupo genético + Idade $(L, Q)+$ touro + erro

2 Modelos: a $\hat{Y}=\mu$ + rebanho-ano-estação + grupo genético + Idade $(L, Q)+P A(L, Q)+P S(L, Q)+$ touro + erro

${ }^{3}$ Mesmo de a + produção total na lactação $(L, Q)$

${ }^{1}$ Model: $\quad \hat{Y}=\mu+$ herd-year-season + breed group + Age $(L, Q)+$ sire + error

${ }^{2}$ Model: $\quad \hat{Y}=\mu+$ herd-year-season + breed group + Age $(L, Q)+P A(L, Q)+P S(L, Q)+$ sire + error

${ }^{3}$ Same as a + total production in the lactation $(L, Q)$

genética para o período seco, por haver correlação genética entre estas características (BROTHERSTONE, 1987). Estas estimativas são semelhantes às encontradas por FUNK et al. (1987), sendo, porém, inferiores às de SCHAEFFER e HENDERSON (1972). Estes valores baixos para heritabilidade sugerem que o período seco e de serviço são características essencialmente influenciadas por variação de meio ambiente e que talvez fosse recomendável o ajustamento das lactações para esses efeitos. Entretanto, tem sido desaconselhável o ajustamento para período seco (BROTHERSTONE, 1987), uma vez que existe correlação genética entre período seco e produção de leite e dificuldade de se precisar se a secagem ocorre naturalmente ou por causa de decisão de manejo. Além disso, o período seco está altamente correlacionado com o intervalo de partos, que não tem apresentado correlação com produção, pelo que o ajustamento para intervalo de partos ou período de serviço removeria maior parte da variação decorrente do período seco.

\section{Conclusões}

A produção de leite aumentou em taxas crescentes com os períodos secos anteriores inferiores a 60 dias, concordando com a recomendação de que este é o período seco ideal.

A estimativa da heritabilidade do período de serviço obtida no presente trabalho foi baixa e muito semelhante às obtidas em vários estudos em que se utilizou o modelo de touro, sugerindo que o período de serviço é efeito de ambiente sobre a produção de leite.

O ajustamento para período de serviço anterior é desejável da mesma forma que o ajustamento para período de serviço na lactação corrente. Embora o valor estimado para a heritabilidade do período seco forneça indicação de ser este, principalmente, efeito de ambiente, o ajustamento para período seco não é recomendável, pois existe correlação genética entre produção e período seco.

\section{Referências Bibliográficas}

AURAN, T., 1974. Studies on monthly and cumulative monthly milk records. II. The effects of calving interval and stage of pregnancy. Acta Agric. Scand., 44(2):339-348.

BAR-ANAN, R., SOLLER, M. 1979. The effects of days-open on milk yield and on breeding policy post partum. Anim. Prod., 29(1):109-119.

BERGER, P. J., SHANKS, R. D., FREEMAN, A. E. et al. 1981. Genetic aspect of milk yield and reprodutive performance. $J$. Dairy Sci., 64(1):114-122.

BOLDMAN, K. G., L. A., KRIESE, L., D., VAN VLECK, C. P. 1995. et al. A manual for use of MTDFREML. A set of programs to obtain estimates of variances and covariances [DRAFT]. Beltsville: U.S. Department of Agriculture, Agricultural Research Service.

BROTHERSTONE, S. 1987. A note on the value and methods of correcting milk records for calving interval on days dry. Anim. Prod., 44(2):322-325.

FUNK, D. A., FREEMAN, A. E., BERGER, P. J. 1987. Effect of previous days open, previous days dry, and present days open on lactation yield. J. Dairy Sci., 70(11):2366-2373.

HANSEN, L. B., FREEMAN, A. E., BERGER, P. J. 1983. Yield and fertility relationships in Dairy Cattle. J. Dairy Sci., 66(2):293-305.

MAKUZA, S. M., McDANIEL, B. T. 1996. Effects of days dry, previous day open, and current days open on milk yields of cows in Zimbabwe and North Carolina. J. Dairy Sci., 79(4):702-709.

MEYER, K. 1987. Restricted maximum likelihood to estimate variance components for mixed models with two random factors. Genet. Sel. Evol., 19(1):49-68.

OLTENACU, P. A., ROUNSAVILLE, T. R., MILLIGAN, R. A. 1980. et al. Relationships between days open and cumulative milk yield at various intervals from parturition for high and low producing cows. J. Dairy Sci., 63(8):1317-1327.

PATTERSON, H. D., THOMPSON, R. 1971. Recovery of interblock information when block sizes are unequal. 
Rev. bras. zootec.

Biometrica, 58(545):554.

SADEK, M. H., FREEMAN, A. E. 1992. Adjustment factors for previous and present days open considering all lactations. $J$. Dairy Sci., 75(1):279-287.

SAS/STAT. User's Guide: statistics Edition, Version 6.04. Cary: SAS Inst., Inc., 1990.

SCHAEFFER, L. R., HENDERSON, G. R. 1972. Effects of days dry and days open on Holstein milk production. J. Dairy Sci., 55(1):107-112.

TEIXEIRA, N. M., FREITAS, A. F., VAlEnTE, J. 1992. Correlações de meio-ambiente entre meio-irmãs paternas para produção de leite na raça holandesa. R. Soc. Bras. Zootec., 21(3):501-509.

THOMPSON, J. R., FREEMAN, A. E., BERGER, P. J. 1982. Days open adjusted, annualized, and fat-corrected yields as alternatives to mature-equivalent records. J. Dairy Sci., 65(8):1562-1577.
VALENTE, J., TEIXEIRA, N. M., VERNEQUE, R. S. et al. Efeitos dos períodos de serviço anterior, período seco anterior e período de serviço corrente sobre a produção de leite. In: REUNIÃO ANUAL DA SOCIEDADE BRASILEIRA DE ZOOTECNIA, 32, 1995, Brasília. Anais... Brasília: SBZ, 1995, p. 686-688.

Recebido em: 03/06/97

Aceito em: 24/08/98 